\title{
Aprender jugando: las formas geométricas y los colores primarios. La Bauhaus y la educación infantil.
}

Natalia Juan natajuan@unizar.es Universidad de Zaragoza, España

Reference

Juan, Natalia; (2012) "Aprender jugando: las formas geométricas y los colores primarios. La Bauhaus y la educación infantil.", p. 126-131. In: Barbosa, Helena; Quental, Joana [Eds]. Proceedings of the 2nd International Conference of Art, Illustration and Visual Culture in Infant and Primary Education. São Paulo: Blucher, 2015

ISSN 2318-695X, ISBN: 978-989-98185-0-7 DOl 10.5151/edupro-aivcipe-25

Este artículo analiza la importancia del juego y cómo éste sirve de herramienta de aprendizaje en la educación infantil. En especial se atiende a los juguetes de construcciones (formas básicas de madera pintadas con los colores primarios) cuyo antecedente histórico lo podemos encontrar en los diseños ideados en 1923 por Alma Siedhoff-Buscher, alumna de la Bauhaus. Este tipo de juguetes, que en la actualidad se denominan "juegos educativos", resultan realmente fructíferos durante el crecimiento así como un poderoso motor de desarrollo personal del niño. Juego, aprendizaje, desarrollo, crecimiento, creatividad, Bauhaus.

Abstract

This paper analyzes the importance of the game and how this is used as a learning tool during the childhood education. Especially we aim to attend to the building/construction toys (basic forms of wood painted with the primary colors) which historical precedent we it can find in the designs devised in 1923 by Alma Siedhoff-Buscher, a Bauhaus' student. This type of toys, which actually are named as "educational games", are really fruitful during the growth as well as a powerful mean of personal development of the child.

\section{Introducción}

\begin{abstract}
"Los niños deben tener un cuarto donde puedan hacer lo que quieran, en el que reinen. Todo lo que hay allí les debe pertenecer, su fantasía lo crea sin ningún impedimento exterior, sin la advertencia '¡Déjalo!'. Todo debe ajustarse a ellos, debe estar a su medida, su utilidad práctica no debe impedir sus posibilidades para el juego. Colores claros y alegres para crear un ambiente divertido y placentero". (Valdivieso, 2007:571)
\end{abstract}

Una declaración de esta índole se comprende en el contexto de la creación de la casa Haus am Horn diseñada por Georg Muche (pintor y profesor de la Bauhaus) en cuyo equipamiento trabajaron los alumnos de los talleres de los que se componía esta escuela. En uno de ellos estaba matriculada Alma Siedhoff-Buscher, alumna de la Bauhaus encargada de la decoración del cuarto infantil de aquella casa, quien escribió esta afirmación en 1926 en un ambiente que -tal y como se deduce- fomentaba el potencial creativo frente a la obediencia ciega y el adoctrinamiento tradicional de la educación infantil de las primeras décadas del siglo XX. Ya entonces -desde los postulados bauhasianos- se pensó en el juego como una actividad que estimulara el desarrollo de los niños, teoría que se mantiene en la actualidad, tal y como vamos a demostrar en este trabajo. 
2nd International Conference Art, Illustration and Visual Culture in Infant and Primary Education $2^{\circ}$ Congreso Internacional

Arte, Ilustración y Cultura Visual en Educación Infantil y Primaria
Congresso Internacional

de Arte, llustracão e Cultura Visual

na Educação Infantil e Primária

\section{El juego en el desarrollo infantil. Aprender de niños, aprender jugando}

El juego es la actividad por excelencia de la infancia, una necesidad básica para los niños porque es algo innato a su propia naturaleza (Gutiérrez, 1997: 11). Es más, se podría decir que es su rasgo más expresivo y definitorio. Jugar resulta de vital importancia para el desarrollo educativo y personal puesto que, a partir del juego, los niños de manera espontánea descubren, observan, exploran, experimentan y comprenden el mundo que tienen a su alrededor que es, en esencia, el mundo de los adultos. Jugando conocen las cosas lo cual favorece el crecimiento y lo potencia (Ancín, 1989).

Jugar es una tarea natural y esencial que supone un estímulo altamente placentero el cual, por otra parte, es imprescindible para el desarrollo integral del niño, ya que está estrechamente relacionado con el crecimiento humano. Así, los niños aprenden a relacionarse con los demás, cultivan sus habilidades, potencian el aprendizaje de los roles sociales, estimulan los sentidos, las facultades del pensamiento, ejercitan la creatividad así como otras capacidades afectivoemocionales y de equilibrio psíquico que les ayudan a crecer con normalidad pues, "el juego conduce de modo natural a la creatividad" (Moyles, 1999: 87) y "ser creativo exige tiempo e imaginación (...) una confianza en sí mismo, cierto conocimiento, receptividad, un sentido del absurdo y la capacidad de jugar, cosas que corresponden muy bien a la niñez y muchas de las cuales necesitan ser engendradas con mayor vigor en el contexto de la escuela y de la educación" (Moyles, 1999: 97). Por todo ello no sólo hay que permitir que los niños jueguen (en su casa y en la escuela) sino que, en la medida de lo posible, hay que fomentarlo y procurar que el juego tenga un fin. El juego es una posibilidad de conocerse a sí mismo, de ampliar horizontes propios, de autoexpresarse o de autodescubrirse, pues jugando llegamos a formar conceptos sobre lo que es nuestro mundo (Spencer, 1976).

El juego debe ser la principal actividad en la vida de los niños desde que son bebés. Jugar no sólo es necesario (tanto como elementos que consideramos básicos como la comida o el abrigo) sino que es vital para lograr un equilibrio dentro de su proceso de desarrollo, aunque todavía hay algunos sectores que se muestran reticentes porque piensan que es sólo una actividad recreativa e ignoran sus propiedades didácticas y educativas. El juego educa, de hecho, desde hace décadas está considerado como el principal medio de aprendizaje para los niños ya que "el juego motiva. Por eso proporciona un clima especial para el aprendizaje" (Moyles, 1999: 43). Hay que tener en cuenta que los niños necesitan constantemente acción y movimiento, tener sensaciones manejando objetos y relacionándose con ellos, aspectos que precisamente consigue el juego de manera natural. Por medio de éste se entretienen, colman su fantasía, disfrutan del goce de crear, consiguen placer y alegría de vivir. El juego les permite expresarse libremente, encauzar sus energías de manera positiva e incluso descargar posibles tensiones infantiles y dominar su ansiedad.

La escuela es el lugar donde se aprenden los conocimientos y habilidades específicas que se consideran útiles tanto para el desarrollo personal como para la integración en la sociedad, ya que, al menos en el llamado mundo desarrollado, todos los niños deben estar escolarizados. El colegio es un medio que les permite socializarse y entrar en contacto con otros niños y adultos, aprendiendo a respetar normas sociales de comportamiento y de convivencia. Hay que tener en cuenta que después del ámbito familiar, el colegio, es el contexto social más inmediato del niño. Por ello, la educación infantil es un periodo del sistema educativo de gran relevancia para el niño pues comienza a relacionarse con sus iguales y empieza a conocer el mundo que les rodea. El juego inicia a los niños en el proceso de aprendizaje del lenguaje, gracias a lo cual adquieren capacidades de comunicación y desarrollan hábitos de atención, de memoria y raciocinio. Mediante estas actividades lúdicas el niño aprende a conocerse a sí mismo, establecer relación con los demás y saber cuáles son sus posibilidades, puesto que le sirve para encontrar su lugar dentro del ámbito social en el que vive.

Uno de los principales objetivos del proceso educativo infantil es el desarrollo de la autonomía personal estimulando el mundo de los afectos. A partir de la realización de variadas actividades y, especialmente, mediante el juego se potencia este progreso personal. En efecto, "el juego es una herramienta que llevada al aula, con unos objetivos claros y precisos, genera un ambiente 
2nd International Conference Art, Illustration and Visual Culture in Infant and Primary Education $2^{\circ}$ Congreso Internacional

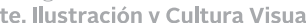
en Educación Infantil y Primaria de Arte, llustração e Cultura Visual

na Educação Infantil e Primária

propicio para que el proceso de aprendizaje sea ameno, efectivo y a la vez productivo" (Nevado, 2008: 1). De hecho, todas las tareas diarias del niño giran en torno a lo lúdico juego, puesto que a través de él, se relacionan con su entorno y forman las bases para el desarrollo de su personalidad (Gutiérrez, 1997: 15). El juego aúna dos elementos básicos y necesarios para el desarrollo del niño. Por un lado, la diversión de la que es plenamente consciente, por lo inmediato y placentero. Por otro, el aprendizaje, aspecto del que no es consciente pero que poco a poco va permeabilizando en su interior, pues "cuando un niño juega, no se trata solamente de una distracción, jugar es hacer trabajar la mente". La infancia es la etapa en la que el niño aprende más y a mayor velocidad, existe en él una necesidad tan fuerte de conocer su entorno que plasma su energía en el incesante quehacer de su actividad diaria. En efecto, el juego es un importante factor que surge de la realidad que rodea al niño y le introduce, sin darse cuenta, en el mundo de las ideas y de la inteligencia humana ya que le proporciona recursos suficientes para participar en diferentes tareas que, normalmente, requieren la presencia de determinados objetos y materiales en un espacio adecuado. El aula es un buen lugar para ello porque el juego entre los niños de la clase permite que éstos establezcan relaciones interpersonales altamente beneficiosas que les provoca un estado de gozo y satisfacción lleno de alegría que les conduce a la felicidad.

Para conseguir todo esto es importante dar libertad al niño para que pueda aprender sin darse cuenta de que lo está haciendo. El juego posibilita que los niños hagan uso de su creatividad y de su imaginación ya que la actividad lúdica capta su interés y su atención. Por eso no resulta acertado interrumpirle o establecerle un orden de cómo debe hacerlo, ni decirle cómo tiene que jugar. No se juega 'bien' o 'mal' y mucho menos desde el criterio del adulto es más "muchos autores han destacado la importancia de incurrir en errores durante el aprendizaje" y el juego es un sistema para ello (Moyles, 1999: 42). Así que, hay que proporcionar libertad al niño pues tiene que seguir su propio camino ya que mientras juega, aprende y establece normas y cada uno -como sabemos- aprende a su ritmo. Los niños deben guiar su propio juego y para ello es fundamental respetar los roles y reglas que asumen mientras juegan, pues sólo permitiéndoles que experimenten y hagan uso de su imaginación podrán desarrollarse plenamente. Buena prueba de ello es que muchas veces no juegan con juguetes sino que utilizan objetos que convierten y transforman en sus propios objetos de juego. Sin embargo, hay que tener en cuenta que un juego en sí mismo no enseña a los niños a actuar. Si bien es recomendable que los padres permitan jugar a los niños, más importante todavía es que jueguen con ellos y "las ludotecas son un medio obvio de lograr que los padres entren en contacto con materiales de juego" (Moyles, 1999: 181). Para ello es fundamental estimular al niño a que aprenda a disfrutar de su tiempo de juego que, en la medida de lo posible, debe compartirse con los progenitores, ya que los juguetes brindan la posibilidad no sólo de educar al niño sino de establecer y consolidar los vínculos afectivos con sus padres mediante el juego.

\section{Los juegos de construcciones: triángulo amarillo, círculo azul y cuadrado rojo}

Los primeros estímulos para captar la atención del niño tienen que ser tan básicos como visuales. Las figuras geométricas constituyen un tema importante en el aprendizaje de los primeros años, ya que están muy ligados a la vida cotidiana y a su desarrollo social. El entorno del niño está lleno de signos, colores y figuras. Por ello, la educación infantil intenta que, desde una edad temprana, pueda adquirir una serie de conocimientos que permita desenvolver al niño en su entorno. Uno de los principales objetivos es introducirle en el desarrollo del pensamiento lógico-matemático partiendo del conocimiento de las figuras geométricas básicas y en el aprendizaje de los colores primarios (Lahora, 2007:162). Estos conceptos se muestran a la perfección en los juegos de construcciones, esto es, aquellos, que están formados por distintas piezas (cuyas formas pueden ser iguales o distintas) con el fin de que el niño las combine formando estructuras. Las piezas permiten una infinita cantidad de posibilidades para construir toda clase de formas reales e imaginarias. Este tipo de juguetes se conforman a partir de elementos que pueden ser bien de pequeño formato para ser usados sobre una mesa o superficie plana, o bien de gran tamaño para jugar directamente en el suelo. Tanto los de un tipo como los de otro enseñan a desarrollar las capacidades motrices de los niños, a manejar los términos grande y pequeño, arriba y abajo, más corto y más largo... Además, con ellos aprenden 
$2^{\text {nd }}$ International Conference Art, Illustration and Visual Culture in Infant and Primary Education
Figuras 1 y 2. A la izquierda: Juguete de madera con las tres formas geométricas básicas y los tres colores primarios según el leit motiv de la Bauhaus conservado en el Museo Museum of Modern Art (MoMA) de Nueva York. A la derecha: Juego de construcción diseñado en 1923 por Alma SiedhoffBuscher. Fuente: propia.

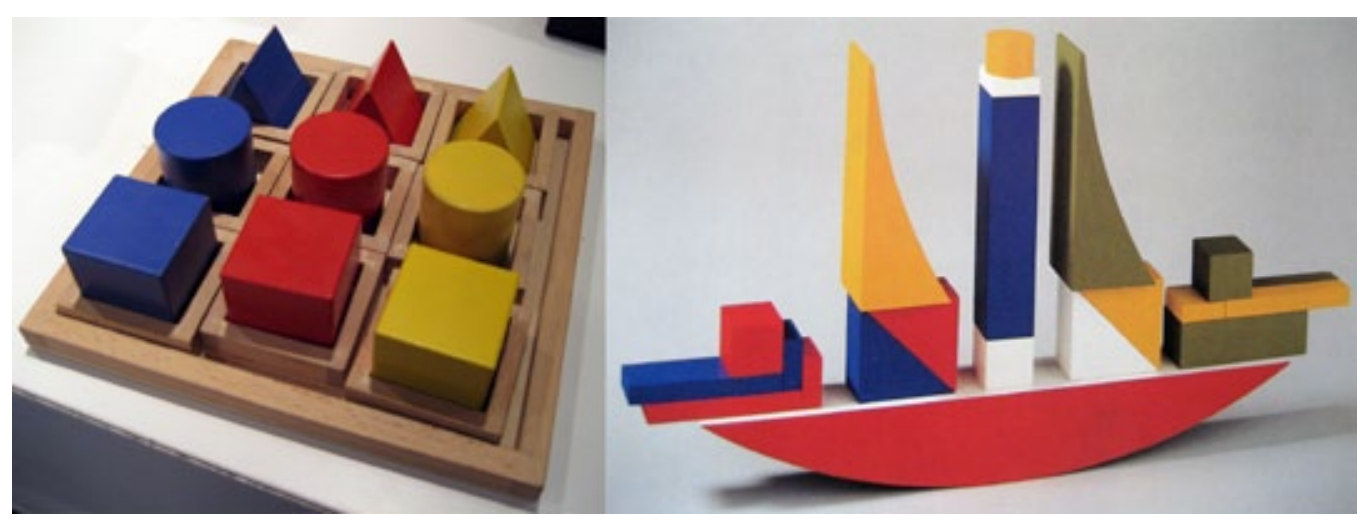

a distinguir entre diversas formas geométricas, a diferenciar los colores, a mejorar su visión espacial y a formarse en nociones básicas sobre el equilibrio, la resistencia o la simetría.

Gracias a los juguetes de construcción los niños aprenden a ordenar, a clasificar las piezas y, si se les enseña, a recoger el material una vez se ha terminado de emplear. Este tipo de juegos resulta útil en todas las edades porque posee diferentes niveles de dificultad y complejidad además, tal y como señalan algunos estudiosos, "en todas las edades se acomete el juego por puro placer y disfrute y engendra una actitud cordial hacia la vida y el aprendizaje" (Moyles, 1999: 21). Así, en el caso concreto de los juegos de construcción, cuando es bebé aprende a cogerlos, golpearlos entre sí o tirarlos desarrollando sus capacidades motrices. Al gatear, transporta las piezas de un lugar a otro o se dedica a apilar, a amontonar o a hacer líneas. Al sentarse llena y vacía las piezas en un recipiente para almacenarlas. Cuando los niños son más mayores, interactúan con las figuras y levantan estructuras que pueden ir desde las más básicas a las relativamente complejas. A los dos años, son capaces de erigir construcciones simples como una fila o una torre vertical y luego derribarlos para después poder comenzar de nuevo. A los tres años, ya pueden realizar torres más complejas y otro tipo de construcciones como casas, puentes o vehículos.

En definitiva, los juegos de construcción se pueden utilizar en diferentes edades y con ellos, los niños aprenden a conocer el medio del que se rodean porque pueden construir los elementos con los que se conforma su espacio urbano y a partir de allí inventar historias relacionadas con lo que ha creado todo lo cual contribuye a desarrollar su capacidad narrativa y descriptiva del mundo en el que vive. En definitiva, los juegos de construcciones contribuyen fundamentalmente a aumentar la coordinación motora. Además, ayudan al razonamiento, al conocimiento de la organización espacial, favorecen la atención, conducen a la reflexión, desarrollan la memoria, potencian la concentración, la paciencia y la capacidad de interpretar unas instrucciones. A través de este tipo de juguetes, los niños aprenden a construir objetos con sus propias ideas y con materiales a su alcance desarrollando su imaginación y su creatividad. Todo esto es lo que debió pensar Alma Siedhoff-Buscher cuando en 1923 ideó el Bauspiel (juego de construcción) durante sus estudios en la Bauhaus. Esta alumna, como la mayoría de estudiantes femeninas que habían ingresado en esta escuela -y a pesar de lo aperturista que resultaba para comienzos del siglo XX- estaba abocada al taller de textil. Buscher vio en el diseño de juguetes para niños una posibilidad para escapar del destino al que había sido irremediablemente guiada.

"Algunos de los maestros de la Bauhaus, como Alma Siedhoff-Buscher, diseñaron sus propios bloques, reconociendo que formas diferentes abrían la puerta a diferentes tipos de composiciones. Una comparación entre los bloques de Siedhoff-Buscher y los llamados Tree Blocks evidencia estas diferencias" (Root-Bernstein. 2002: 267).

El contraste entre un juego y otro es tan notable que salta a primera vista lo que indica que se dirigen a finalidades diferentes. En efecto, el Bauspiel ideado por Buscher es un juego compuesto por 22 bloques de madera cuyos diversos colores, formas y dimensiones permiten jugar con la imaginación y construir todo tipo de figuras (sin embargo, las piezas de los Tree 
$2^{\text {nd }}$ International Conference Art, Illustration and Visual Culture in Infant and Primary Education
Figura 4. Juego de la serie Mula de Ikea compuesto por 24 piezas de madera. [Consulta 10 mayo 2007]. Disponible en <http://wwwikea.com/es/es/catalog/ products $/ 80028929 />$
Blocks no están pintadas y son únicamente rectángulos y cilindros con una clara alusión a los troncos de los árboles). El diseño del Bauspiel debe entenderse como uno de los resultados del programa pedagógico de la Bauhaus ya que la relación entre la fabricación artística, la producción técnica-seriada y sus dimensiones sociales fue una de las máximas filosóficas de esta escuela de diseño. El objetivo del Bauspiel era conseguir que el niño fuese capaz de distinguir una forma geométrica determinada de otra. Además, Buscher pretendía que el niño pudiese reconocer las tres figuras geométricas básicas, los colores primarios, clasificar los bloques lógicos de acuerdo con su forma, color y tamaño así como desarrollar su capacidad de observación, propósitos que se persiguen hoy en día en las escuelas de educación infantil. Por ello, debemos considerar que las enseñanzas en relación a la forma y al color desarrolladas actualmente en las aulas de infantil ya fueron puestas en práctica en el diseño de juguetes de la Bauhaus de donde proviene un lejano eco.
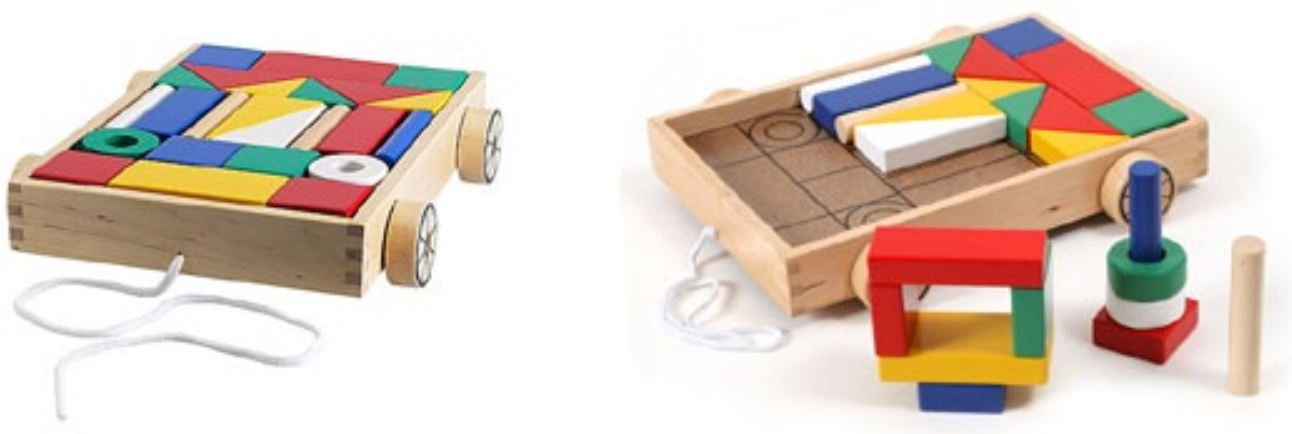

Aunque, sin duda alguna, los diseños de la Bauhaus que directamente se han transmitido en la educación infantil son los juguetes ideados por la alumna, Alma Siedhoff-Buscher. En la actualidad, los niños aprenden con este juego de construcción (que todavía se vende) porque sus básicas formas geométricas y su vivo colorido despiertan la imaginación y la creatividad. En efecto, la fábrica suiza Naef Spiele compró la licencia de los diseños de la Bauhaus en los años 60 del siglo XX y por ello está autorizada a hacer réplicas de los juguetes diseñador por Buscher a partir de los dibujos originales guardados en los archivos berlineses de esta importante escuela. Desde 1977 Naef Spiele tiene autorizado no sólo la producción de la réplica del Bauspiel sino también de la caja que lo contiene, todo un clásico del diseño en el sentido verdadero de la palabra. La versión popular es el juego de la serie Mula de lkea compuesto por 24 piezas de madera de diferentes formas geométricas entre las que hay cubos, rectángulos, triángulos o cilindros de diversos colores (rojo, azul, amarillo y verde) que desarrollan las habilidades motrices y el pensamiento lógico. Las piezas se insertan en una estructura con ruedas que hace las veces de carromato el cual lleva una plantilla de colocación en el fondo del carrito que permite recoger las piezas de manera más fácil. De esta manera, los niños de 3 a 6 años son capaces de distinguir las formas geométricas y sus diferentes cualidades cromáticas gracias a la herencia que nos dejó la Bauhaus, cuyo valioso legado se mantiene hoy vivo entre los más pequeños. Una última reflexión cabe señalar aquí, si el juego Bauspiel funcionó (y funciona) bien como estímulo creativo para los niños, el Mula de lkea que es en esencia lo mismo, aunque con pequeñas variaciones ¿cumple con idéntica función aunque no tengan el marchamo de "diseño de calidad" que si tiene todo producto relacionado con la escuela de la Bauhaus? El desarrollo de la respuesta afirmativa a esta pregunta será tratada en posteriores trabajos a los que nos dedicaremos en un futuro. 
$2^{\text {nd }}$ International Conference Art, Illustration and Visual Culture in Infant and Primary Education $2^{\circ}$ Congresso Internacional

de Arte, Ilustração e Cultura Visual

na Educação Infantil e Primária

\section{Referencias bibliográficas}

Ancín, M.T., (1989). Cuerpo, espacio, lenguaje. Madrid: Ed. Narcea.

Arranz, J.D., (1995). Juegos al aire libre. Educación Infantil y Primaria. Madrid: Ed. Escuela Española. Bitterberg, K.G., (1976). Bauhaus. Stuttgart: Institut für Auslandsbeziehungen.

Blanco Sierra, J., (1992). El juego infantil. Orense: Editorial Juan XXIII.

Droste, M., (1998). Bauhaus 1919-1933. Berlin: Bauhaus Archiv.

Garaigardobil, M., (1990). Juego y desarrollo infantil. Madrid: Editorial Seco Olea.

Glanzer, M., (2000). El juego en la niñez. Un estudio de la cultura lúdica infantil. Buenos Aires: Aique.

Gutiérrez, R., (1997). El juego de grupo como elemento educativo. Madrid: Ed. CCS.

Kandinsky, W., (1983). Cursos de la Bauhaus. Madrid: Alianza Editorial, 1975.

Kandinsky, W., (1983). De lo espiritual en el arte. Barcelona: Editorial Barral, 1912.

Kandinsky, W., (2003). Punto y línea sobre el plano. Buenos Aires, Paidós, 1926.

Lahora, C., (2007). Actividades matemáticas con niños de o a 6 años. Barcelona: Narcea

María A. G. de Granato et al., (1992). El juego en el proceso de aprendizaje. Buenos Aires: Humanitas.

Moyles, J.R. (1999). El juego en la educación infantil y primaria. Madrid: Ediciones Morata.

Nevado, CH., (2008). "El componente lúdico en las clases de ELE”. Revista de Didáctica ELE, n 7, 2008. [http://marcoele.com/el-componente-ludico-en-las-clases-de-ele/]

Pérez C., (ed.) (2010). Los juguetes de las vanguardias. Málaga: catálogo de la Exposición del Museo Picasso de Málaga.

Oppenheim, J.F., (1990). Los juegos Infantiles. Barcelona: Ed. Martínez Roca.

Ortega, R., (1990). El juego infantil y la construcción social del conocimiento. Sevilla Edición Alfar.

Root-Bernstein, R. y Root-Bernstein M., (2002), El secreto de la creatividad. Barcelona: Kairós.

Piaget, J., (1977). Psicología de las edades: del nacer al morir. Madrid: Morata.

Piaget, J., (1982). Los años postergados: la primera infancia. Barcelona: Paidós.

Spencer, Z.A., (1976). 150 Juegos y actividades Preescolares. Barcelona: Ed. CEAC.

Vigotski, L. S., (2001). Psicología pedagógica: Un curso breve. Buenos Aires: Aique.

Valdivieso, M., (2007). "La aportación de la Bauhaus a la innovación del espacio doméstico: la 'casa modelo' Haus am Horn (1923)", VV.AA., Espacios Interiores. Casa y arte desde el siglo XVIII al XXI, Barcelona, Universitat de Barcelona, Centro de Recherches Historiques sur les Sociétés Méditerranéennes, Université de Perpignan: 565-575.

Wick, R., (1998). Pedagogía de la Bauhaus. Madrid: Alianza.

Will, C. (1998). Alma Siedhoff-Buscher. Köln: Buchhandlung Walther Köning. 